07

\title{
Адаптивный фотоприемник на основе эффекта нестационарной фото-ЭДС в задачах регистрации упругих деформаций и напряжений
}

\author{
( М.А. Брюшинин, ${ }^{1}$ В.В. Куликов, ${ }^{1}$ А.А. Петров, ${ }^{1}$ И.А. Соколов, ${ }^{1}$ А.М. Балбашов ${ }^{2}$ \\ ${ }^{1}$ Физико-технический институт им. А.Ф. Иофрфе РАН, \\ 194021 Санкт-Петербург, Россия \\ ${ }^{2}$ Московский энергетический институт, \\ 111250 Москва, Россия \\ e-mail: mb@mail.ioffe.ru
}

(Поступило в Редакцию 14 апреля 2016 г.)

Исследовано детектирование оптических фазомодулированных сигналов, создаваемых механическими колебаниями прозрачного объекта - стеклянной плоскопараллельной пластины. Регистрация сигнала осуществлялась с помощью интерферометрической схемы, использующей эффект нестационарной фото-ЭДС в адаптивном фотоприемнике на основе кристалла $\beta-\mathrm{Ga}_{2} \mathrm{O}_{3}$. Механическая система, состоящая из стеклянной пластины и пьезоэлектрического преобразователя, демонстрирует резонансное поведение в области частот $100 \mathrm{kHz}$. Распределение амплитуды фазовой модуляции по поверхности пластины имеет колоколообразный профиль в выбранной области частот. Характеристики адаптивного фотоприемника определены для длины волны света $\lambda=532 \mathrm{~nm}$.

DOI: $10.21883 /$ JTF.2017.03.44247.1932

\section{1. Введение}

За последние годы возникло довольно большое количество интерферометрических приложений, требующих регистрации отклика среды на колебательное воздействие [1-7]. Использование фоторефрактивных кристаллов в таких приложениях обеспечивает достаточно высокую чувствительность, возможность работы со сложными волновыми фронтами, а также адаптивность интерферометра. Перечисленные характеристики могут быть также достигнуты и в схеме, использующей адаптивный фотоприемник на основе эффекта нестационарной фото-ЭДС.

Эффект нестационарной фото-ЭДС заключается в возникновении переменного электрического тока в образце, освещаемом колеблющейся интерференционной картиной. Ток появляется вследствие периодических пространственных сдвигов решеток фотопроводимости и поля пространственного заряда. В формировании этих решеток участвуют процессы возбуждения носителей заряда, диффузии и дрейфа в электрическом поле, а также захвата на локальные центры. Такой сложный характер возникновения сигнала позволяет использовать эффект для исследования фотоэлектрических процессов и определения параметров материала, таких как тип и величина проводимости, время жизни, диффузионная длина и подвижность носителей заряда [8-12]. Другим применением эффекта является детектирование оптических фазо- и частотно-модулированных сигналов [13-19].

Детектирование оптических фазомодулированных сигналов с использованием адаптивных фотоприемников обычно проводится в виброметрической схеме, в которой фазовая модуляция создается путем отражения света от колеблющегося объекта. В настоящей работе мы исследуем режим возбуждения сигнала нестационарной фото-ЭДС, в котором фазовая модуляция сигнального луча возникает при прохождении света через прозрачный объект, подвергающийся периодической упругой деформации. В качестве прозрачного объекта выбрана плоскопараллельная стеклянная пластина ПМ-15, а в качестве адаптивного фотоприемника впервые используется кристалл $\beta-\mathrm{Ga}_{2} \mathrm{O}_{3}$, приобретающий все большую популярность в современной оптике и электронике [20-24].

\section{2. Экспериментальная установка}

Установка для исследования упругих колебаний прозрачного объекта показана на рис. 1. Свет второй гармоники $\mathrm{Nd}$ :YAG-лазера с длиной волны $\lambda=532 \mathrm{~nm}$ разделяется на два луча с мощностями $P_{r}=4 \mathrm{~mW}$ и $P_{s}=6 \mathrm{~mW}$. Эти лучи формируют интерференционную картину с пространственной частотой $K$, контрастом $m=0.98$ и средней интенсивностью $I_{0}$ на поверхности кристалла. Один из лучей проходит через прозрачную стеклянную пластину ПМ-15. Пластина изготовлена из стекла К8 и имеет диаметр $30.3 \mathrm{~mm}$ и толщину $15.87 \mathrm{~mm}$. Передняя и задняя поверхности имеют оптическое качество. На боковой цилиндрической поверхности присутствуют две диаметрально расположенные плоские площадки (лыски) шириной $6.6 \mathrm{~mm}$, используемые для зажима пластины. Колебательная деформация пластины создается пьезоэлектрическим преобразователем. Для калибровки фотоприемника на основе $\beta-\mathrm{Ga}_{2} \mathrm{O}_{3}$ используется электрооптический модулятор, размещенный в опорном луче интерферометра. Будучи включенным он вносит фазовую модуляцию с амплитудой $\Delta_{c}=0.61$ и частотой $\omega$. Ток, возникающий в фотоприемнике, 


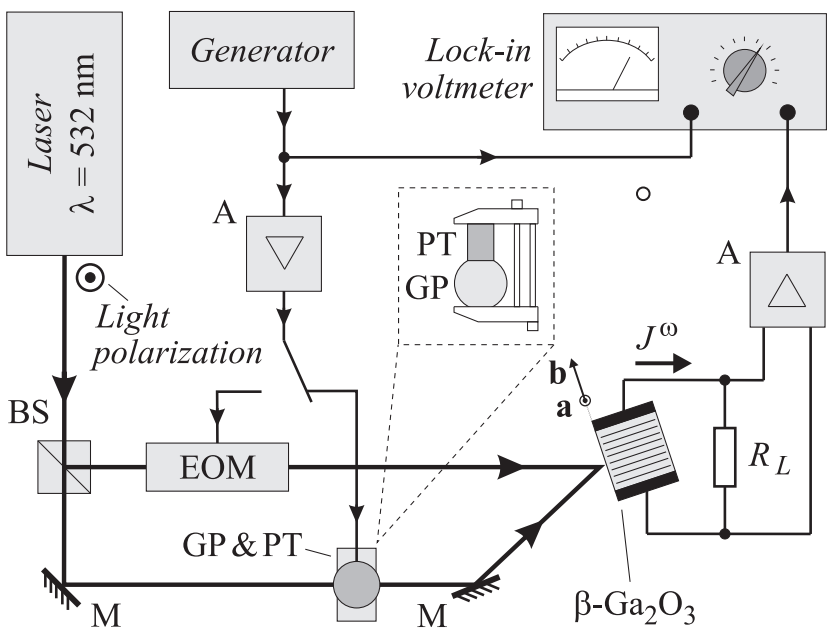

Рис. 1. Экспериментальная установка для исследования механических колебаний прозрачных объектов. ЕОМ - электрооптический модулятор, BS - светоделитель, М - зеркало, А усилитель, GP - стеклянная пластина, РТ - пьезоэлектрический преобразователь. На вставке, обозначенной штриховой линией, изображены стеклянная пластина, пьезоэлектрический преобразователь и сжимающая их струбцина в плоскости, перпендикулярной направлению распространения света.

приводит к появлению напряжения на нагрузочном сопротивлении, которое затем усиливается и измеряется lock-in вольтметром. Плоскость поляризации перпендикулярна плоскости падения света на фотоприемник (ТЕполяризация).

$\beta-\mathrm{Ga}_{2} \mathrm{O}_{3}$ является моноклинным кристаллом с размерами элементарной ячейки $a=1.223 \mathrm{~nm}, b=0.304 \mathrm{~nm}$, $c=0.580 \mathrm{~nm}$ и $\beta=103.7^{\circ}[25]$. Ширина запрещенной зоны составляет $E_{g}=4.84 \mathrm{eV}$ [26]. Статическая диэлектрическая проницаемость является тензором с главными значениями $\epsilon_{11}=10.84, \epsilon_{22}=11.49$ и $\epsilon_{33}=13.89$ [27].

Кристалл оксида галлия был выращен методом зонной плавки на установке URN-2-ZM, собранной в НИУ МЭИ [28]. Затравка ориентировалась так, чтобы рост осуществлялся вдоль направления [010]. Выбранная скорость роста в $10 \mathrm{~mm} / \mathrm{h}$ обеспечивала стабильность процесса кристаллизации. Качество получаемых монокристаллов зависит от типа атмосферы и ее давления. Образец, используемый в настоящей работе, был выращен в кислородной атмосфере с давлением $60 \mathrm{bar}$, обеспечившей наилучшее качество кристалла.

Исследование выращенного кристалла методами рентгеновской и оптической спектроскопии было проведено в предыдущей работе [29]. Используя метод Лауэ, кристалл был сориентирован, были подтверждены параметры решетки, известные из литературы [25], а также показано отсутствие двойникования кристалла. Кристалл демонстрирует пропускание порядка $80 \%$ в видимой и инфракрасной областях спектра $(400-1100 \mathrm{~nm})$ и край поглощения в области $\lambda<270 \mathrm{~nm}$, соответствующий межзонным переходам.
Исследуемый образец $\beta-\mathrm{Ga}_{2} \mathrm{O}_{3}$ имеет размеры $2.00 \times 2.15 \times 1.35 \mathrm{~mm}$ вдоль кристаллографических направлений [100], [010] и вдоль направления, перпендикулярного плоскости (001). Передняя и задняя поверхности $(2.00 \times 2.15 \mathrm{~mm})$ получены сколом кристалла вдоль плоскости (001), никакая дополнительная обработка поверхностей не применялась. С использованием серебряной пасты были нанесены электроды на боковые грани (010).

\section{3. Экспериментальные результаты}

Характерная амплитуда сигнала нестационарной фото-ЭДС в кристалле $\beta$ - $\mathrm{Ga}_{2} \mathrm{O}_{3}$ составляет $10^{-12}-10^{-9} \mathrm{~A}$, что примерно на два порядка меньше значений, наблюдаемых в кристаллах $\mathrm{Bi}_{12} \mathrm{Si}(\mathrm{Ti}, \mathrm{Ge}) \mathrm{O}_{20}[8,30]$. Тем не менее такая величина достаточна для детектирования с отношением сигнал/шум 0-60 dB. Фаза нестационарной фото-ЭДС соответствует электронному типу проводимости материала.

Для калибровки адаптивного фотоприемника была снята амплитудно-частотная характеристика нестационарной фото-ЭДС в кристалле $\beta-\mathrm{Ga}_{2} \mathrm{O}_{3}$ (рис. 2), т.е. была измерена зависимость $J^{\omega}=J^{\omega}\left(\Delta_{c}, \omega\right)$ при постоянной амплитуде фазовой модуляции $\Delta_{c}$. На зависимости присутствует участок линейного роста сигнала на низких частотах с последующим выходом на частотнонезависимый участок. Рост сигнала в области низких частот является проявлением адаптивности процесса формирования пространственного заряда в фотопроводящих материалах [8,31]. В этой области частот сигнал мал вследствие того, что решетка поля пространственного заряда и решетка свободных носителей (решетка фотопроводимости) отслеживают движения интерференционной картины. Пространственный сдвиг между решетками поддерживается примерно равным $\pi / 2$, что и приводит

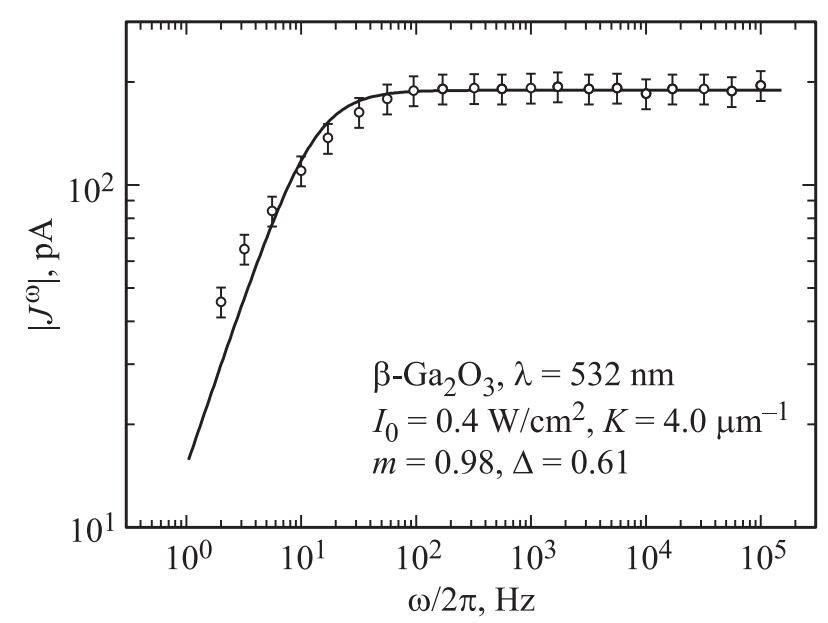

Рис. 2. Амплитудно-частотная характеристика нестационарной фото-ЭДС в адаптивном фотоприемнике на основе кристалла $\beta-\mathrm{Ga}_{2} \mathrm{O}_{3}$. Сплошной линией показана аппроксимация выражением (1). 


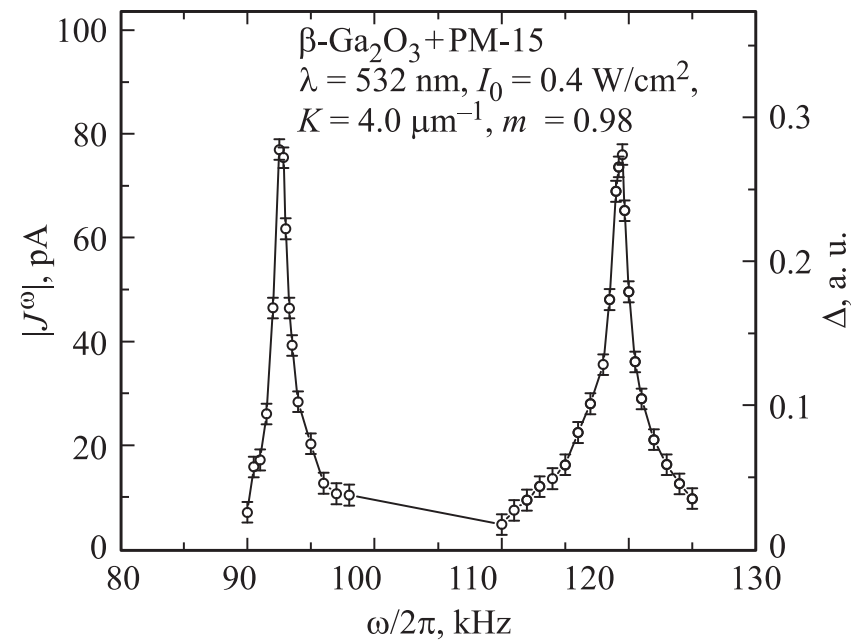

Pис. 3. Частотная зависимость нестационарной фото-ЭДС и соответствующей амплитуды фазовой модуляции при возбуждении сигнала светом, прошедшим через колеблющуюся стеклянную пластину ПМ-15.

к малой величине сигнала. При более высоких частотах решетка с бо́льшим временем релаксации становится практически неподвижной, периодические пространственные сдвиги решеток увеличиваются, и ток достигает максимума (частотно-независимый участок). Так как на зависимости нет спадающего участка, формирование пространственного заряда происходит в условиях квазистационарной фотопроводимости $(\omega \tau \ll 1)$, а сама зависимость описывается довольно простым выражением $[8,32]$

$$
J^{\omega}=S^{2} \mathrm{~J}_{0}(\Delta) \mathrm{J}_{1}(\Delta) \sigma_{0} E_{D} \frac{-i \omega \tau_{M}}{1+i \omega \tau_{M}\left(1+K^{2} L_{D}^{2}\right)} .
$$

Здесь $E_{D}=\left(k_{B} T / e\right) K$ - диффузионное поле, $\tau_{M}=$ $=\epsilon_{0} \epsilon / \sigma_{0}-$ время максвелловской релаксации, $L_{D}-$ диффузионная длина электронов, $\mathrm{J}_{n}-$ функция Бесселя первого рода $n$-го порядка, $S$ - площадь электродов, $k_{B}$ - постоянная Больцмана, $T-$ температура, $e-$ элементарный заряд, $\epsilon_{0}-$ электрическая постоянная. Участок линейного роста и плато на частотной зависимости разделены частотой среза $\omega_{1}$ :

$$
\omega_{1}=\left[\tau_{M}\left(1+K^{2} L_{D}^{2}\right)\right]^{-1} .
$$

Для зависимости, представленной на рис. 2, эта частота среза составляет $13 \mathrm{~Hz}$, что соответствует следующим значениям времени максвелловской релаксации и фотопроводимости: $\tau_{M}=10 \mathrm{~ms}, \sigma_{0}=$ $=1.0 \cdot 10^{-10} \Omega^{-1} \mathrm{~cm}^{-1}[30]$. Следует отметить, что на частотной зависимости нет второго частотно-независимого участка и соответствующих частот среза, характерных для полупроводников, в которых перенос заряда сопровождается захватом на мелкие ловушки $[11,12,32]$.

После калибровки фотоприемника можно перейти к исследованию упругих колебаний в прозрачном объекте. Частотная зависимость нестационарной фото-ЭДС, возбуждаемой колебаниями стеклянной пластины, представлена на рис. 3. В общем случае такая зависимость включает в себя как частотный отклик исследуемого объекта, так и детекторную характеристику: $J^{\omega}=$ $=J^{\omega}(\Delta(\omega), \omega)$. Амплитуда фазовой модуляции $\Delta(\omega)$, создаваемой механическим колебаниям объекта, рассчитывается из следующего нелинейного уравнения:

$$
\frac{\mathrm{J}_{0}(\Delta(\omega)) \mathrm{J}_{1}(\Delta(\omega))}{\mathrm{J}_{0}\left(\Delta_{c}\right) \mathrm{J}_{1}\left(\Delta_{c}\right)}=\frac{J^{\omega}(\Delta(\omega), \omega)}{J^{\omega}\left(\Delta_{c}, \omega\right)} .
$$

B наших экспериментах со стеклянной пластиной амплитуда фазовой модуляции сравнительно мала $(\Delta<0.3)$, а выбранный диапазон частот $90-125 \mathrm{kHz}$ соответствует плато на частотной характеристике фотоприемника на основе $\beta-\mathrm{Ga}_{2} \mathrm{O}_{3}$. Это позволяет линеаризовать уравнение (3), так, что расчет искомой частотной зависимости $\Delta(\omega)$ сводится к умножению измеренной зависимости $J^{\omega}(\Delta(\omega), \omega)$ на некоторый коэффициент или к добавлению еще одной оси на график, как это было сделано нами на рис. 3. Резонансное поведение измеряемого сигнала $J^{\omega}$, очевидно, обусловлено резонансом механической системы, включающей в себя стеклянную пластину, пьезоэлектрический преобразователь и струбцину, используемую для их зажима. Увеличение силы, прижимающей пластину и преобразователь друг к другу, немного увеличивает резонансные частоты.

Так как стеклянная пластина имеет достаточно большие размеры и сложную форму, можно ожидать, что механические напряжения, деформации и определяемая ими амплитуда фазовой модуляции неоднородно распределены по поверхности. Мы измерили это распределение, сдвигая струбцину с зажатыми пластиной и преобразователем в направлениях, перпендикулярных лучу света (рис. 4). Сканирование осуществлялось с шагом $2.4 \mathrm{~mm}$ по обеим координатам, всего было снято 103 точки. Измерения проводились при $\omega / 2 \pi=93.5 \mathrm{kHz}$, т. е. на первой резонансной частоте. Полученное распределение обладает примерно радиальной симметрией с максимумом, находящимся в центре пластины. Наблюдаемый

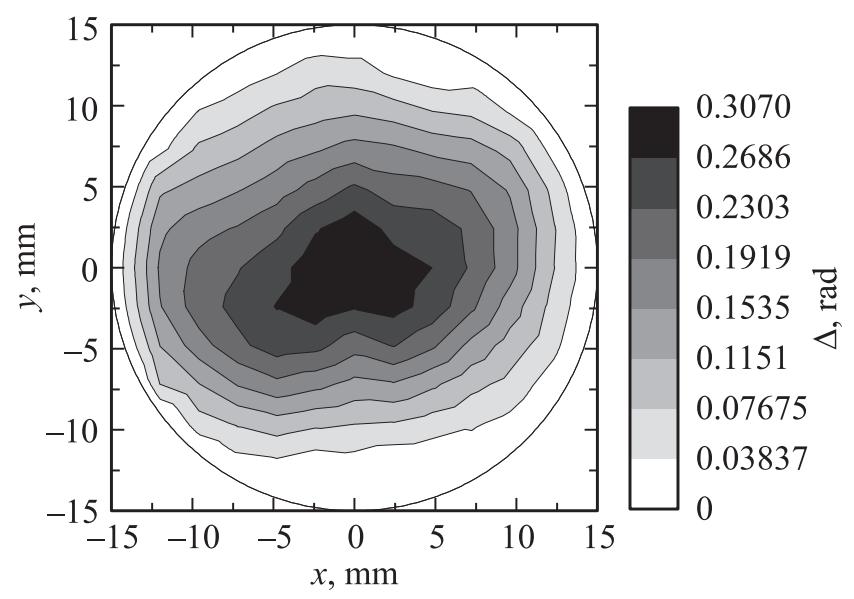

Рис. 4. Распределение амплитуды фазовой модуляции по поверхности стеклянной пластины ПМ-15. 
небольшой наклон распределения, вероятно, обусловлен неоднородностью прикладываемой силы по поверхности плоской площадки.

\section{4. Обсуждение результатов}

В настоящей работе мы использовали перспективный полупроводниковый кристалл $\beta-\mathrm{Ga}_{2} \mathrm{O}_{3}$ как материал для изготовления адаптивного фотоприемника оптических фазомодулированных сигналов. Такой выбор не вполне очевиден: сигнал нестационарной фотоЭДС возбуждается светом с длиной волны $\lambda=532 \mathrm{~nm}$ и энергией фотона $h v=2.33 \mathrm{eV}$ при том, что ширина запрещенной зоны кристалла составляет $4.8 \mathrm{eV}$. В связи с этим возникает вопрос о чувствительности фотоприемника для выбранных условий эксперимента. Мы определяем чувствительность адаптивного фотоприемника как амплитуду сигнала, нормированную на мощность сигнального луча и амплитуду фазовой модуляции: $S^{\omega}=J^{\omega} /\left(P_{s} \Delta\right)=1.5 \cdot 10^{-7} \mathrm{~A} \cdot \mathrm{W}^{-1} \mathrm{rad}^{-1}$. Шумы в нашей измерительной системе в основном определяются тепловыми шумами нагрузочного сопротивления $R_{L}=300 \mathrm{k} \Omega$. Отсюда следует, что минимальная детектируемая амплитуда фазовой модуляции равна $\Delta_{\min }=\left(4 k_{B} T R_{L}^{-1} \delta f\right)^{1 / 2} /\left(S^{\omega} P_{s}\right)$, где $\delta f-$ полоса детектирования. Для типичных значений $P_{s}=1 \mathrm{~mW}$ и $\delta f=1 \mathrm{~Hz}$ эта величина составляет $\Delta_{\min }=1.6 \cdot 10^{-3} \mathrm{rad}$. Эта оценка уступает значениям, полученным ранее для других широкозонных полупроводников [15], но тем не менее позволяет говорить о высокой чувствительности интерферометра, использующего данный фотоприемник.

Для расчета набега фазы света в общепринятых голографических методах [33] используется подсчет светлых и темных полос на интерференционной картине. Фазовый сдвиг в соседних светлых и темных полосах равен $\pi$, что намного превышает минимальную детектируемую амплитуду исследуемого фотоприемника. Лучшая чувствительность адаптивного фотоприемника, главным образом, обусловлена узкой полосой детектирования, которая имеет порядок величины $1 \mathrm{~Hz}$ и может быть уменьшена до $0.01 \mathrm{~Hz}$ и менее при использовании современных lock-in вольтметров. Однако есть и недостаток разработанной методики по сравнению с классической интерферометрией: в наших экспериментах распределение фазы регистрировалось путем поточечных измерений, тогда как в традиционных методах аналогичное распределение фиксируется целиком.

В настоящей работе мы ограничились определением величины амплитуды фазовой модуляции, возникающей вследствие колебательных упругих деформаций и напряжений в стекле. Известно, что дополнительный набег фазы света возникает за счет пьезооптического эффекта и за счет удлинения/сжатия образца. Процедура расчета пьезооптического эффекта известна из литературы [34] и включает в себя вычисление тензора напряжений $\sigma_{k l}$ в кристаллографической системе координат, вычисление тензора диэлектрической непроницаемости $\xi_{i j}=\pi_{i j k l} \sigma_{k l}$ в кристаллографической системе координат $\left(\pi_{i j k l}-\right.$ тензор пьезооптических коэффициентов), расчет тензора $\xi_{i j}$ в специальной системе координат, определение направлений собственных колебаний для электромагнитных волн и вычисление соответствующих приращений фазы света. Удлинение образца вдоль направления распространения света определяется компонентами тензора деформаций. Так как тензоры упругих деформаций и напряжений связаны друг с другом посредством закона Гука, дополнительный набег фазы света полностью определяется полем механических напряжений (деформаций). Различают две задачи: вычисление компонент тензора пьезооптических коэффициентов по известному полю напряжений в кристалле [35] и расчет напряжений в веществе с известными свойствами [33]. В зависимости от выбора задачи может потребоваться измерение двух и более распределений фазы, аналогичных представленному на рис. 4 , для различных направлений и состояний поляризации света. Решение подобных обратных задач рассеяния является довольно обширной областью исследований [36] и выходит за рамки настоящей работы, где мы постарались показать принципиальную возможность использования адаптивных фотоприемников для регистрации фазомодулированных оптических сигналов, возникающих при прохождении света через объект, подвергающийся периодическому механическому воздействию.

\section{5. Заключение}

В настоящей работе осуществлено детектирование оптических фазомодулированных сигналов, создаваемых упругими колебаниями прозрачного объекта. Измерена амплитудно-частотная характеристика адаптивного фотоприемника на основе кристалла $\beta-\mathrm{Ga}_{2} \mathrm{O}_{3}$, определена его чувствительность $S^{\omega}=1.5 \cdot 10^{-7} \mathrm{~A} \cdot \mathrm{W}^{-1} \mathrm{rad}^{-1}$, а также минимальная детектируемая амплитуда фазовой модуляции $\Delta_{\min }=1.6 \cdot 10^{-3} \mathrm{rad}$ (для $P_{s}=1 \mathrm{~mW}$ и $\delta f=1 \mathrm{~Hz}$ ). Колебания механической системы, состоящей из стеклянной пластины и пьезоэлектрического преобразователя, демонстрируют резонансное поведение в окрестности $100 \mathrm{kHz}$. Измеренное пространственное распределение амплитуды фазовой модуляции имеет колоколообразный профиль с максимумом, находящимся в центре пластины.

Работа выполнена при поддержке Российского научного фонда (грант 15-12-00027).

\section{Список литературы}

[1] Kamshilin A.A., Romashko R.V., Kulchin Y.N. // J. Appl. Phys. 2009. Vol. 105. N 3. P. 031101.

[2] Yu P., Nolte D.D., Melloch M.R. // Opt. Lett. 2003. Vol. 28. N 10. P. $819-821$. 
[3] Murray T.W., Sui L., Maguluri G., Roy R.A., Nieva A., Blonigen F., DiMarzio C.A. // Opt. Lett. 2004. Vol. 29. N 21. P. 2509-2511.

[4] Колегов А.А., Шандаров С.М., Симонова Г.В., Кабанова Л.А., Буримов Н.И., Шмаков С.С., Быков В.И., Каргин Ю.Ф. // Квант. электрон. 2011. Т. 41. Вып. 9. C. $847-852$.

[5] Шандаров С.М., Шмаков С.С., Буримов Н.И., Сюваева О.С., Каргин Ю.Ф., Петров В.М. // Письма в ЖЭТФ. 2012. Т. 95. Вып. 12. С. 699-702.

[6] Ромашко Р.В., Ебимов Т.А., Кульчин Ю.Н. // Квант. электрон. 2014. Т. 44. Вып. 3. С. 269-273.

[7] Romashko R.V., Kulchin Y.N., Nippolainen E. // Laser Phys. 2014. Vol. 24. N 11. P. 115604.

[8] Petrov M.P., Sokolov I.A., Stepanov S.I., Trofimov G.S. // J. Appl. Phys. 1990. Vol. 68. N 5. P. 2216-2225.

[9] Sokolov I.A., Bryushinin M.A., Ordin S.V., Kulikov V.V., Petrov A.A. // J. Phys. D: Appl. Phys. 2006. Vol. 39. N 6. P. 1063-1068.

[10] Bryushinin M., Kulikov V., Mokhov E., Nagalyuk S., Sokolov I. // Phys. Rev. B. 2012. Vol. 86. N 8. P. 085209.

[11] Bryushinin M.A., Kulikov V.V., Mokrushina E.V., Mokhov E.N., Petrov A.A., Sokolov I.A. // J. Phys. D: Appl. Phys. 2014. Vol. 47. N 41. P. 415102.

[12] Брюшинин М.А., Петров А.А., Писарев Р.В., Соколов И.А. // ФТТ. 2015. Т. 57. Вып. 5. С. 892-897.

[13] Stepanov S.I., Sokolov I.A., Trofimov G.S., Vlad V.I., Popa D., Apostol I. // Opt. Lett. 1990. Vol. 15. N. 21. P. 1239-1241.

[14] Брюшинин М.А., Куликов В.В., Соколов И.А. // ЖТФ. 2002. Т. 72. Вып. 10. С. 79-86.

[15] Bryushinin M.A., Grattan K.T.V., Kulikov V.V., Sokolov I.A. // J. Mod. Opt. 2006. Vol. 53. N. 5-6. P. 857-864.

[16] Bryushinin M., Kulikov V., Sokolov I., Delaye P., Pauliat G. // EPL (Europhys. Lett.). 2014. Vol. 105. N 6. P. 64003.

[17] Bryusinin M., Kulikov V., Sokolov I., Delaye P., Pauliat G. // J. Opt. Soc. Am. B. 2014. Vol. 31. N 4. P. 723-729.

[18] Bryushinin M.A., Kulikov V.V., Mokhov E.N., Romashko R.V., Sokolov I.A. // Optik. 2016. Vol. 127. N 1. P. 341-344.

[19] Брюшинин М.А., Соколов И.А., Завестовская И.Н., Кульчин Ю.Н. // Кр. сообщ. физ. 2015. Т. 42. Вып. 12. С. 97-100.

[20] Orita M., Ohta H., Hirano M., Hosono H. // Appl. Phys. Lett. 2000. Vol. 77. N 25. P. 4166.

[21] Feng P., Zhang J.Y., Li Q.H., Wang T.H. // Appl. Phys. Lett. 2006. Vol. 88. N 15. P. 153107.

[22] Oshima T., Okuno T., Arai N., Suzuki N., Ohira S., Fujita S. // Appl. Phys. Express. 2008. Vol. 1. N 1. P. 011202.

[23] Higashiwaki M., Sasaki K., Kuramata A., Masui T., Yamakoshi S. // Appl. Phys. Lett. 2012. Vol. 100. N. 1. P. 013504.

[24] Guo D.Y., Wu Z.P., An Y.H., Li P.G., Wang P.C., Chu X.L., Guo X.C., Zhi Y.S., Lei M., Li L.H., Tang W.H. // Appl. Phys. Lett. 2015. Vol. 106. N 4. P. 042105.

[25] Geller S. // J. Chem. Phys. 1960. Vol. 33. N 3. P. 676-684.

[26] Peelaers H., Van de Walle C.G. // Phys. Stat. Sol. B. 2015. Vol. 252. N 4. P. 828-832.

[27] Liu B., Gu M., Liu X. // Appl. Phys. Lett. 2007. Vol. 91. N 17. P. 172102.

[28] Balbashov A.M., Egorov S.K. // J. Cryst. Growth. 1981. Vol. 52. N 2. P. 498-504.

[29] Voronchikhina M.E., Balbashov A.M., Galagan B.I., Shulman I.L. In: Scientific Program and Book of Abstracts: 17th International Conference on Crystal Growth and Epitaxy. Warsaw, Poland, August 11-16, 2013. P. 379-380.
[30] Bryushinin M.A., Sokolov I.A., Pisarev R.V., Balbashov A.M. // Opt. Express. 2015. Vol. 23. N 25. P. 32736-32 746.

[31] Петров М.П., Степанов С.И., Хоменко А.В. Фоторефрактивные кристаллы в когерентной оптике. СПб.: Наука, 1992. $320 \mathrm{c}$.

[32] Bryushinin M.A., Sokolov I.A. // Phys. Rev. B. 2000. Vol. 62. N 11. P. 7186-7194.

[33] Вест Ч. Голографическая интерферометрия. М.: Мир, $1982.504 \mathrm{c}$.

[34] Сиротин Ю.И., Шаскольская М.П. Основы кристаллофизики. М.: Наука, 1975. 680 с.

[35] Krupych O., Savaryn V., Vlokh R. // Appl. Opt. 2014. Vol. 53. N 10. P. B1-B7.

[36] Sweeney D.W., Vest C.M. // Appl. Opt. 1973. Vol. 12. N 11. P. 2649-2664. 